\title{
Human-based fibrillar nanocomposite hydrogel as bioinstructive matrices to tune stem cell behavior
}

Received 00th January 20xx Accepted 00th January $20 x x$ DOI: $10.1039 / x 0 x \times 00000 x$

\author{
Bárbara B. Mendes $^{a, b, c}$, Manuel Gómez-Florit ${ }^{a, b, c}$, Ricardo A. Pires ${ }^{a, b, c}$, Rui M. A. Domingues ${ }^{a, b, c} *$, Rui \\ L. Reis $^{a, b, c}$, Manuela E. Gomes ${ }^{a, b, c *}$
}

The extracellular matrix (ECM)-biomimetic fibrillar structure of platelet lysate (PL) gels along with its enriched milieu of biomolecules has drawn significant interest in regenerative medicine applications. However, PL-based gels have poor structural stability which severely limits its performance as a bioinstructive biomaterial. Here, rod-shaped cellulose nanocrystals (CNC) are used as a novel approach to modulate the physical and biochemical microenvironment of PL gels enabling their effective use as injectable human-based cell scaffolds with a level of biomimicry that is difficult to recreate with synthetic biomaterials. The incorporation of CNC ( 0 to $0.61 \mathrm{wt} . \%$ ) into the PL fibrillar network during the coagulation cascade leads to decreased fiber branching, increased interfiber porosity (from 66 to 83\%) and modulate fiber (from $1.4 \pm$ 0.7 to $27 \pm 12 \mathrm{kPa}$ ) and bulk hydrogel (from $18 \pm 4$ to $1256 \pm 82 \mathrm{~Pa}$ ) mechanical properties. As result of these physicochemical alterations, nanocomposite PL hydrogels resist to the typical extensive clot retraction (from $76 \pm 1$ to $24 \pm$ 3 at Day 7) and show favored retention of PL bioactive molecules. The feedback of these cues on the fate of human adipose-derived stem cells is evaluated, showing how it can be explored to modulate the commitment of encapsulated stem cells toward different genetic phenotypes without the need for additional external biological stimuli. These fibrillar nanocomposite hydrogels allow therefore to explore the outstanding biological properties of human-based PL as an efficient engineered ECM which can be tailored to trigger specific regenerative pathways in minimal invasive strategies.

\section{Introduction}

Blood components orchestrate the highly complex microenvironment present in wound healing by providing a wide range of biological, chemical and scaffold-forming structural elements $^{1,2}$. In particular, platelet-rich blood derivatives contain an enriched milieu of growth factors (GFs), cytokines, and temporary extracellular matrix (ECM) precursors (e.g. fibrinogen and vitronectin) that have shown to influence stem cell behavior ${ }^{3-6}$. Among them, platelet lysate (PL) is particularly interesting because it shows a comparatively lower batch-to-batch variability due to its preparation method based on platelet disruption instead of activation with calcium or/and thrombin (e.g. platelet-rich fibrin), which clearly contributes to standardization of formulations ${ }^{7-10}$, and

\footnotetext{
3B's Research Group, I3Bs - Research Institute on Biomaterials, Biodegradables and Biomimetics, University of Minho, Headquarters of the European Institute of Excellence on Tissue Engineering and Regenerative Medicine, Avepark - Parque de Ciência e Tecnologia, Zona Industrial da Gandra, 4805-017 Barco - Guimarães, Portugal.

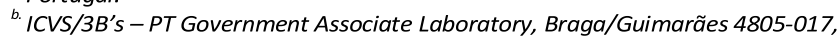
Portugal

The Discoveries Centre for Regenerative and Precision Medicine, Headquarters at University of Minho, Avepark, 4805-017 Barco, Guimarães, Portugal

*Corresponding author: megomes@i3bs.uminho.pt,

rui.domingues@i3bs.uminho.pt

Electronic Supplementary Information (ESI) available: Materials and methods details. Morphological and chemical characterization of modified CNC and additional physical hydrogel characterization. See: DOI: 10.1039/x0xx00000x
}

because it hardly forms a spontaneous gel due to the removal of clot and platelet debris during its preparation that allows an easy further processing ${ }^{10}$.

In clinical settings, the delivery of platelet-rich blood derivatives relies on their direct injection and further spontaneous platelet activation upon contact with the native collagen tissue ${ }^{11,12}$, or on the induction of the coagulation cascade prior to their injection by the addition of thrombin and/or calcium to be used as a fibrin-based scaffold ${ }^{13}$. Interestingly, the produced fibrin scaffold is organized into a ECM mimetic fibrillar network that contains key cell adhesion binding motifs, which promote an efficient tissue healing ${ }^{14}$. Despite their rapid translation into clinical applications for the treatment of numerous conditions, particularly of the musculoskeletal system ${ }^{3}$, several pre-clinical studies and clinical trials have continuously shown contradictory therapeutic outcomes $^{15,16}$. Among the several limitations that can be identified to justify these results, there is the lack of well-designed clinical trials, the poor characterization of blood derivatives samples and the lack of control over the delivery mechanisms of multiple signalling biomolecules ${ }^{10}$. Moreover, current natural fibrillar gel scaffolds solely based on blood derivatives components exhibit limited ability to retain and protect biological active biomolecules from degradation ${ }^{17}$, have extremely low mechanical properties and structural stability ${ }^{18}$, and show a high contractile effect $(\sim 75 \%$ reduction in gel volume) upon cell encapsulation, severely limiting their potential as cell carriers and modulators of wound healing ${ }^{19}$. 
The reinforcement of low strength matrices generated by chemically or physically crosslinking natural and synthetic polymers with nanomaterials is being explored as tissue engineering and regenerative (TERM) strategies to produce a new generation of nanocomposite hydrogels with added functionalities (e.g. electrical or magnetic responsiveness) ${ }^{20,21}$, as well as to modulate their mechanical and/or biological properties, or to trigger specific cellular responses ${ }^{22}$. In this sense, we and others have been using cellulose nanocrystals (CNC), i.e. rod-shaped nanoparticles produced from cellulose fibers, as natural-based nanofillers to improve the mechanical, structural and cell supporting properties of soft hydrogel matrices ${ }^{23-25}$. The outstanding strength and stiffness, high aspect ratio, convenient surface chemistry and high biocompatibility of $\mathrm{CNC}$, are some of the properties that make them an appealing alternative to other typical reinforcing nanofillers. Additionally, the presence of sulfate groups on CNC surface resulting from their typical production method (based on sulfuric acid hydrolysis) might potentially allow an efficient binding of GFs through specific and reversible electrostatic interactions. This process would mimic the natural stabilization of GFs by sulfated glycosaminoglycans (GAGs) present in the ECM that are known to regulate their spatio-temporal presentation and signaling mechanisms in native tissues ${ }^{26,27}$. In fact, it has been demonstrated that soluble cellulose sulfate derivatives with structural similarities to heparin, an highly sulfated GAG, establish specific interactions with growth factors, namely BMP-2 and FGF-2 ${ }^{28-30}$, suggesting that the sulfate groups on CNC surface might play a relevant role on the active modulations of the hydrogel biological microenvironment. In addition, CNC reinforcement might enable to tune the mechanical properties of fibrin network at the fiber level in order to stabilize the natural fibrillar nature of blood derivatives and to use them as injectable biomimetic scaffolding biomaterials.

In this work, we developed an injectable and multifunctional nanocomposite fibrillar hydrogel based on the induction of $\mathrm{PL}$ coagulation cascade while incorporating CNC modified with surface aldehyde groups (see supplementary information Figure S1). Conceptually, CNC will be entrapped in the fibrils' structure and simultaneously crosslinked through reversible Schiff base bonds established with the amine groups of PL proteins. Thus, it is anticipated that this simple one-pot polymerization and crosslinking method potentiates its fast in situ gelation and structural stabilization after extrusion while allowing their administration using minimal invasive strategies. We hypothesize that the incorporation of CNC will not only have a positive impact on the microstructural and mechanical properties of the fibrillar structure, but will also enable to modulate the biochemical microenvironment generated within the hydrogel. It is expected that these dynamic alterations on the physicochemical properties of PL-based fibrillar hydrogels can influence the biological behavior of encapsulated stem cells and enable their use in a wide range of TERM applications.

\section{Experimental}

Hydrogels production. Stock solutions of $\mathrm{PL}$ and a-CNC were produced and fully characterized (for details on the preparation and characterization, see supporting information). Double-barrel syringe L-system (1:1 from Medmix, Switzerland) with a static mixer tip (Medmix, Switzerland) was used to produce the hydrogels. Barrel A was filled with PL (67.6 mg mL $\mathrm{mL}^{-1}$ of total protein) composed of albumin, growth factors, cytokines and structural proteins (such as fibrinogen, vitronectin and fibronectin). Barrel B was composed of thrombin from human plasma ( $2 \mathrm{U} \mathrm{mL}^{-1}$, Sigma-Aldrich, USA), calcium (10 mM, Sigma-Aldrich, USA) and a-CNC water dispersions corresponding to $0,2.3,4.5,9$ and 18 wt.\% of PL total protein content. The precursor solutions were then hand extruded into cylindrical acrylic molds ( $10 \mathrm{~mm}$ diameter $\times 6 \mathrm{~mm}$ height). Finally, the samples were incubated during 2 hours at $37{ }^{\circ} \mathrm{C}$ to allow PL matrix polymerization and crosslinking. The final hydrogels were all composed of 50 vol.\% of PL and were named according to their aCNC concentrations: 0 wt.\% (PL-CNC 0), 0.15 wt.\% (PL-CNC 0.15), 0.31 wt.\% (PL-CNC 0.31), 0.45 wt.\% (PL-CNC 0.45), and 0.61 wt.\% (PL-CNC 0.61).

\section{Characterization of nanocomposite hydrogel microstructure.} Hydrogels were produced as described above and then fixed in $2 \%$ glutaraldehyde (Merck, Germany) for 2 hours. After fixation, samples were solvent exchanged from water to ethanol (ethanol gradient from 25, 50, 75 and 99.9 vol.\%) for 4 hours and then critical point dried with $\mathrm{CO}_{2}$. After immersion in liquid nitrogen, the samples were freeze-fractured to expose their inner structures, and sputter coated (30 seconds at $20 \mathrm{~mA}$, Cressington) with gold prior observation in a scanning electron microscope (JSM-6010LV, JEOL, Japan). Based on the typical fibrinogen concentration range in $\mathrm{PL}$ $\left(454 \pm 75 \mathrm{\mu g} \mathrm{mL}^{-1}\right)^{31}, 9 \mu \mathrm{g}$ of Alexa Fluor ${ }^{\circledast} 488$ conjugated fibrinogen from human plasma (Thermo Fisher Scientific, USA) were added to each $1 \mathrm{~mL}$ of PL solution (barrel A) for visualization of the fibrin network by confocal microscopy. For this analysis, PL-CNC hydrogels were produced in PDMS molds (9 $\mathrm{mm}$ diameter $\times 2 \mathrm{~mm}$ height) on a glass coverslip and observed on a laser scanning confocal TCS SP8 (Leica Microsystems, Germany) with a 63x immersion objective. Twenty micrometer thick Z-stacks were imaged at every $0.2 \mu \mathrm{m}$ beginning $5 \mu \mathrm{m}$ above the coverslip surface. PL-CNC hydrogels porosity was calculated using ImageJ software (National Institutes of Health, USA). Z-stacks obtained from confocal fluorescence were subdivided into $4 \mu \mathrm{m}$ Z-stacks (20 frames) and the corresponding projected images were filtered with Gaussian Blur filter (Sigma radius 1), background subtracted (pixel 15) and sharpen. From these filtered images, a thresholding mask was set to the mean pixel intensity of the image and the image was inverted to determine the percent area that was not considered as a fiber. Then, the porosity area was determined indirectly.

Polymerization kinetics and mechanical properties of nanocomposite hydrogels. A Kinexus Prot Rheometer (Malvern Instruments, United Kingdom) with a parallel-plate geometry and a hood for temperature control $\left(37^{\circ} \mathrm{C}\right)$ was used to measure the 
viscoelastic properties of PL-CNC hydrogels. Time-sweep tests were applied to study polymerization kinetics of the different formulations. Immediately after dispensing $320 \mu \mathrm{L}$ of extruded solution on the bottom plate, a $20 \mathrm{~mm}$ diameter top plate was lowered to a final gap of $1 \mathrm{~mm}$ (parallel plate set up). A thin film of mineral oil was poured over the exposed surface to reduce water evaporation. This time point was defined as the starting time $(\mathrm{t}=0)$. Time-sweep assays were performed at a strain amplitude of $1 \%$ and frequency of $1 \mathrm{~Hz}$, and storage modulus values were monitored continuously at 10 seconds intervals. In strain and frequencysweeps tests, hydrogels were formulated as described above and an $8 \mathrm{~mm}$ top plate was used (parallel plate set up). A fixed frequency of $1 \mathrm{~Hz}$ was applied with a strain amplitude from 0.01 to $100 \%$ to establish the linear region. At a fixed strain amplitude value $(0.5 \%)$ selected within linear viscoelastic region, frequency sweeps from 0.1 to $10 \mathrm{~Hz}$ were performed to obtain the frequency dependence of the storage modulus $\left(G^{\prime}\right)$ and loss modulus $\left(G^{\prime \prime}\right)$. All measurements were performed in triplicate. Nanomechanical properties of the fibrillary hydrogels were assessed by AFM (NanoWizard 3, JPK Instruments, Germany). Hydrogels were prepared on a coverslip sample holder (JPK Instruments) and kept hydrated in PBS. Cantilevers (Bruker, MSNL-10-C, nominal k $=0.01$ $\mathrm{N} \mathrm{m}^{-1}$ ) was pre-calibrated in PBS using a contact-based method (on a glass surface). Height and moduli images ( $256 \times 256$ pixels) were acquired over a $100 \mu \mathrm{m}^{2}$ square area of the hydrogel. Moduli were calculated by fitting the results to the Sneddon Model using a Poisson ratio of 0.5 . At each acquired point it was used an indentation depth between 0.5-1.0 $\mu \mathrm{m}$. JPK SPM Data Processing (JPK Instruments) were used to determine hydrogel $(n=4)$ and fiber $(n=20)$ rigidity.

Hydrogel Degradation. For degradation assays, hydrogels were produced in a circular polypropylene supports $(9 \mathrm{~mm}$ diameter $\mathrm{x} 4$ $\mathrm{mm}$ height). Each support was pre-weighted, and their weight was subtracted to the total weight of the support with hydrogel to obtain the hydrogel initial mass (Mi). After 2 hours of incubation at $37{ }^{\circ} \mathrm{C}$ to allow hydrogel polymerization and crosslinking, each hydrogel was incubated in $1.5 \mathrm{~mL}$ of PBS pH=7.4 at $37{ }^{\circ} \mathrm{C}$ and successively weighted at different time points to obtain the final mass (Mf). The weight loss was calculated according to the following Equation 1. The results are an average of three measurements obtained per formulation $(n=4)$.

Weight loss $(\%)=\frac{\mathrm{Mi}-\mathrm{Mf}}{\mathrm{Mi}} \times 100$

(Equation 1)

Growth factor release. Hydrogels were produced in circular supports $(\mathrm{V}=0.4 \mathrm{~mL})$ as described above for hydrogel degradation assay and incubated in $0.7 \mathrm{~mL}$ of cell culture medium at $37 \stackrel{\circ}{\circ}$. At different time points $(6 \mathrm{~h}, 1,2,4,5$ days), the supernatant was collected and replaced with fresh cell culture medium. PlateletDerived Growth Factor-BB (PDGF-BB) content in the supernatant of PL-CNC 0, 0.31 and $0.61(n=5)$ was quantified using human PDGF-BB enzyme-linked immunosorbent assay (ELISA) Development kit (Peprotech, USA) according to the manufacturer's instructions. Furthermore, on day 5 , remaining hydrogels were disrupted using a tissue grinder (Nippon Genetics, Japan) and 5 seconds of sonication using a sonication probe. After centrifugation, the pellet was used to detect PDGF-BB by sodium dodecyl sulfate-polyacrylamide gel electrophoresis (SDS-PAGE) and Western Blot. Laemmli sample buffer was mixed with standard PDGF-BB (Peprotech, USA) at 1:1 ratio, and with hydrogels pellet and $\mathrm{PL}$ at 9:1 ratio. All formulations were heated at $95{ }^{\circ} \mathrm{C}$ for 5 minutes and subjected to SDS-PAGE using $3 \%$ stacking and $12.5 \%$ separation polyacrylamide gels prepared according to manufacturer's protocol (SDS Gel Preparation kit, Sigma Aldrich, USA). Electrophoresis was carried out at $75 \mathrm{~V}$ for $15 \mathrm{~min}$ and then at $150 \mathrm{~V}$ for $1 \mathrm{~h}$ (BioRad, USA). The SDS-PAGE and polyvinylidene difluoride membrane were overlapped and proteins transferred at $70 \mathrm{~V}$ and $350 \mathrm{~mA}$ for $15 \mathrm{~min}$ (Pierce Power station, Thermo Fisher Scientific, USA). 5\% milk powder solution was used for membrane blocking for $30 \mathrm{~min}$ at RT. The membrane was then incubated with 1:100 dilution rabbit antiPDGF-BB (Peprotech, USA) primary antibody under mild mixing at $4{ }^{\circ} \mathrm{C}$ overnight. The membrane was washed with TBS-T solution for $5 \mathrm{~min}(3 \mathrm{x})$ then incubated with a 1:1000 dilution of goat anti-rabbit IgG - alkaline phosphatase (Vector laboratories, USA) mixed at RT for 30 minutes, before washing the membrane as described above. The alkaline phosphatase conjugate substrate (Thermo Fisher Scientific, USA) was added to the polyvinylidene difluoride membrane until color development, and after water washing, membranes were scanned.

Cell isolation and encapsulation in the hydrogels. Human adiposederived stem cells (hASCs) were obtained from lipoaspirate samples of the abdominal region of patients undergoing plastic surgery, under the scope of previously established protocols with Hospital da Prelada (Porto, Portugal) with the approval of the Hospital Ethics Committee and informed consent was obtained from all human subjects. The hASCs isolation and stemness characterization were performed using a previous optimized ${ }^{32}$ Briefly, the lipoaspirate samples were washed with PBS and $1 \%$ antibiotic/antimycotic solution until erythrocytes removal, and then digested with $0.05 \%$ collagenase Type I A in PBS for 60 minutes at $37 \stackrel{\circ}{\circ} \mathrm{C}$ under gentle stirring. The digested tissue was filtered and centrifuged at $800 \mathrm{xg}$ for 10 minutes at $4 \stackrel{\circ}{ } \mathrm{C}$ and the supernatant removed. The heterogeneous cell pellet was resuspended in $\alpha$-MEM medium supplemented (Thermo Fisher Scientific, USA) with $10 \%$ fetal bovine serum (FBS) (Thermo Fisher Scientific, USA), and $1 \%$ antibiotic/antimycotic solution (Thermo Fisher Scientific, USA). After 24 hours of incubation, the adherent cells were washed (hASCs) and characterized by flow cytometry for the expression of mesenchymal stem cells markers (CD45, CD105 and CD90), Figure S4, as previously reported to define multipotent mesenchymal stromal cells ${ }^{33-35}$. hASCs were maintained in $\alpha$-MEM supplemented with $10 \% \mathrm{FBS}$, and $1 \%$ antibiotic/antimicotic solution at 37 ㅇ, $5 \%$ $\mathrm{CO}_{2}$. hASCs were loaded to PL solution (barrel B) at a density of $2 \times 10^{6}$ cells per $1 \mathrm{~mL}$ of $\mathrm{PL}\left(1 \times 10^{6}\right.$ cells per $1 \mathrm{~mL}$ of final hydrogel), then hydrogels were produced as described above. All cell culture assays of hASCs encapsulated in hydrogels were performed using culture media without FBS. 
Cellular viability and proliferation studies. Cellular viability was assessed using 1:500 vol.\% Calcein AM (Thermo Fisher Scientific, USA) and 1:1000 vol.\% propidium iodide (Thermo Fisher Scientific, USA) for $15 \mathrm{~min}$ at $37{ }^{\circ} \mathrm{C}$ to stain live and dead cells, respectively. Samples were washed with phosphate buffered saline (PBS) to reduce background fluorescence and visualized using confocal microscope TCS SP8 (Leica Microsystems, Germany). The metabolic activity of encapsulated hASCs was evaluated at 1, 4 and 7 days using Alamar Blue assay (Bio-Rad, USA). Fluorescence readings of Alamar Blue were normalized to the deoxyribonucleic acid (DNA) content. For DNA quantification, hydrogels were sonicated three times for $10 \mathrm{~s}$ using an ultrasound probe at $40 \%$ of amplitude output. Released DNA was quantified using Quant-iT PicoGreen dsDNA assay kit (Thermo Fisher Scientific, USA) following manufacturer's instructions.

Cell morphology and matrix production. hASCs encapsulated in all PL-CNC hydrogels were cultured up to -8 days. Additionally, to analyze cell morphology within the fibrillar network, some hydrogel formulations were supplemented with fibrinogen-FITC, as described above for the analysis of the hydrogel microstructure. Hydrogels were washed with PBS and then fixed in 10\% formalin (Thermo Fisher Scientific, USA) for $15 \mathrm{~min}$ at RT and permeabilized using 0.2 vol.\% Triton-X100 (Sigma-Aldrich, USA). After washing, samples were incubated with $1: 200 \mathrm{v} / \mathrm{v}$ rhodamine-conjugated phalloidin (Sigma-Aldrich, USA) for 10 minutes and 1:1000 v/v 4', 6-diamidino2-phenylindole (DAPI, Sigma-Aldrich, USA) for 20 minutes (dilutions in PBS). For collagen type III, samples were blocked using $3 \mathrm{w} / \mathrm{v} . \%$ BSA and incubated with $1: 100 \mathrm{v} / \mathrm{v}$ solution of specific primary antibody (ab7778, Abcam, UK). Then, samples were incubated with 1:200 v/v Alexa Fluor ${ }^{\circledR} 488$ conjugated secondary antibody (Thermo Fisher Scientific, USA). The hydrogels were observed under a confocal microscope TCS SP8 (Leica Microsystems, Germany). The effect of CNC content on cell spreading area and cell axial ratio was characterized by analyzing their shape using Image J software object tools. Fluorescence confocal images were converted to binary images to identify individual cells and a mean threshold was used to quantify morphometric parameters. Aspect ratio and cell area were quantified at day 1 for all formulations $(n=20)$ and at day $3(n=20)$ for PL-CNC 0.61 formulation.

Ribonucleic acid (RNA) isolation and real-time polymerase chain reaction (PCR) analysis. After 1 and 7 days of cell culture, total RNA was isolated using Ribozol (Amresco, USA), according to manufacturer's protocol. Total RNA was quantified at $260 \mathrm{~nm}$ using a nanodrop spectrophotometer (Thermo Fisher Scientific, USA). The same amount of RNA $(0.2 \mu \mathrm{g})$ was reverse transcribed to complementary DNA (cDNA) according to the protocol of the supplier (qScript cDNA Synthesis Kit, Quanta Biosciences, USA). Aliquots of each $c D N A$ sample were frozen $\left(-20^{\circ} \mathrm{C}\right)$ until the PCR reactions were carried out. Real-time PCR was performed for two reference genes, glyceraldehyde-3-phosphate dehydrogenase (GAPDH) and $\beta$-Actin and target genes (Table S1). Real-time PCR was performed in a thermocycler (Realplex, Eppendorf, Germany) using SYBR green detection (Quanta Biosciences, USA). Each reaction contained $7 \mu \mathrm{l}$ of master mix (Perfecta SYBR Green FastMix, Quanta Biosciences, USA), the sense and the antisense specific primers $(0.5 \mu \mathrm{M})$ and cDNA sample $(3 \mu \mathrm{l})$ in a final volume of $10 \mu \mathrm{l}$. The amplification program consisted of a pre-incubation step for denaturation of the template cDNA ( 5 minutes $95{ }^{\circ} \mathrm{C}$ ), followed by 45 cycles consisting of a denaturation step (10 seconds $\left.95^{\circ} \mathrm{C}\right)$, an annealing step $\left(10\right.$ seconds $60^{\circ} \mathrm{C}$ ) and an extension step (10 seconds $72{ }^{\circ} \mathrm{C}$ ). After each cycle, fluorescence was measured at $72{ }^{\circ} \mathrm{C}$. A negative control without cDNA template was run in each assay. All samples were normalized by the geometric mean of the expression levels of $\beta$-Actin and GAPDH and fold changes were related to the control groups using the equation 2 adapted from ${ }^{36}$.

ratio $=\frac{\mathrm{E}_{\text {target }}^{\Delta \mathrm{Cp} \text { target (mean control - sample) }}}{\mathrm{E}_{\text {reference }}^{\Delta \text { Cp reference (mean control - sample) }}}$

(Equation 2)

$\mathrm{Cp}$ is the crossing point of the reaction amplification curve and $\mathrm{E}$ is the efficiency from the given slopes using serial dilutions. Stability of reference genes was calculated using a statistical tool (BestKeeper software, Technical University of Munich, Weihenstephan, Germany) $)^{37}$.

Alkaline phosphatase activity. After 9 days of culture, hydrogels were washed with PBS and cells were lysed in water using two freeze-thaw cycles, mechanical disruption and ultrasounds. ALP activity from $25 \mu \mathrm{l}$ of sample was quantified by measuring the cleavage of $100 \mu \mathrm{l}$ of p-Nitrophenyl phosphate (pNPP; SigmaAldrich, USA) in a soluble yellow end product that absorbs at 405 $\mathrm{nm}$. In parallel, a standard curve with calf intestinal ALP (Roche, Germany) was constructed. Readings were normalized to the DNA content, determined as described above.

Statistical Analysis. The statistical analysis of data was performed using GraphPad PRISM v 7.0. One-way or two-way analysis of variance (ANOVA) was used to analyze experimental data, followed by Tukey posthoc test for multiple comparisons. Statistical significance was set to $p<0.05$. Results are presented as mean \pm standard deviation.

\section{Results \& Discussion}

CNC content tuned the microstructural and physicochemical properties of fibrillar nanocomposite hydrogels. Fibrin gels result from the self-assembly of fibrinogen induced by thrombin and/or calcium ions, being widely explored as a multifunctional framework for cell adhesion, proliferation and differentiation ${ }^{13}$. In our study, the nanocomposite hydrogel was extruded using a double-barrel syringe (ratio 1:1). Barrel A was filled with PL $\left(67.6 \mathrm{mg}^{-\mathrm{mL}^{-1}}\right.$ of total protein content) and barrel $B$ was composed of thrombin, calcium $\left(\mathrm{CaCl}_{2}\right)$ and a-CNC water dispersions. Herein, the concentrations of thrombin and calcium were pre-optimized to maximize the hydrogel mechanical properties and stability (see supplementary information Fig. S2). The final concentration of $1 \mathrm{U} \cdot \mathrm{mL}^{-1}$ thrombin 
and $5 \mathrm{mM} \mathrm{CaCl}_{2}$ produced relatively fast gelling and stable $\mathrm{PL}$ gel clots and was therefore chosen as the control condition (PL-CNC 0). CNC were then incorporated at varying concentrations (0.31-1.22 wt.\%) to obtain nanocomposite fibrillar hydrogels with the final concentration of 0.15-0.61 wt.\% (PL-CNC 0.15-0.61) (Scheme 1).

The impact of CNC on individual fiber characteristics and hydrogel network microstructure were analyzed by confocal and scanning electron microscopy (SEM), Figure 1. For confocal microscopy analysis, $9 \mu \mathrm{g}$ of fluorescently labeled fibrinogen was added to $1 \mathrm{~mL}$ of PL solution in order to enable the observation of the fiber structure. Similarly to the previously reported characteristic of other PL based hydrogel ${ }^{31}, \mathrm{PL}-\mathrm{CNC} 0$ exhibited a dense meshwork of highly branched and randomly oriented fibrin fibers with low interfiber space (Figure 1-A and D). The incorporation of $\mathrm{CNC}$ led to an overall decrease of fiber branching and gradual increase of interfiber porosity from $66 \%$ in PL-CNC 0 up to $83 \%$ in PL-CNC 0.61 (Figure 1-B). Interestingly, the same trend is also observed in fiber diameter, with PL-CNC 0 having relatively thin fibers (152 $\pm 54 \mathrm{~nm}$ ) that gradually become thicker with increasing CNC incorporation in nanocomposite hydrogels $(259 \pm 85 \mathrm{~nm}$ in PLCNC 0.61), Figure 1-C.

Under physiological conditions, fibrin central domains interact nonconvalently with complementary sites at the end domains of other fibrin molecules and assemble into two-stranded protofibrils. Upon growing to a sufficient length, protofibrils start to laterally associate ${ }^{38-40}$. In our study, the observed physical differences demonstrate that CNC have an impact on the fibrinogen selfassembly and fibrin network architecture mechanisms. Considering CNC dimensions ( $3.5 \pm 1.3 \mathrm{~nm}$ of height and $168 \pm 60 \mathrm{~nm}$ of length, determined by atomic force microscopy), it is plausible that CNC might get entrapped along the fibers during the lateral aggregation of fibrin protofibrils, and at the same time introduce intermolecular covalent bonds that might further promote this process. Since the aldehyde groups on CNC's surface allow them to act as covalent protein crosslinkers, it potentiate the natural fiber packing, playing a similar role as Factor XIIla, which forms crosslinks between the protofibrils within each fiber and later complete the maturation of the network structure ${ }^{41,42}$. Consequently, fibers gradually become more densely packed with increasing CNC content, ultimately resulting in thicker and straighter fibers as observed in the nanocomposite hydrogels.

The structural differences induced by the incorporation of CNC in PL hydrogels led to significant changes in their physical properties. Figure 2 shows the mechanical properties at different length scales (bulk and nanoscale), degradation behavior and platelet-derived GF-BB (PDGF-BB) release profile of hydrogels prepared with different CNC concentrations (0 to 0.61 wt.\%). Concerning the bulk mechanical behavior, no significant differences could be detected for gelation time, with all formulations showing a solid-like behavior $\left(G^{\prime}>G^{\prime \prime}\right)$ right after extrusion $(t=0)$ rather than behaving as a fluid (Figure 2-A). Nevertheless, increasing CNC loadings gradually increased the storage modulus $\left(G^{\prime}\right)$ plateau, although at the highest CNC content it could be noticed a slower progression that did not fully cease after 1.5 hour. This behavior finds some parallelism with the slow increase of stiffness observed in blood clots along the time, a process governed by Factor XIIIa ${ }^{41}$, supporting our hypothesis on the effect of CNC over the mechanisms of fiber formation. In general, all formulations showed a relatively fast polymerization and crosslinking, reaching a near $\mathrm{G}^{\prime}$ plateau within 5 to $10 \mathrm{~min}$ of incubation, demonstrating therefore its potential to be used as an injectable and in situ crosslinkable formulation.

Measurements within the hydrogel linear viscoelastic region demonstrated that bulk hydrogel stiffness significantly increased compared to the neat PL matrix, even at the low CNC loadings used here (Figure 2-B). While PL-CNC 0 had a low storage modulus (18 \pm $4 \mathrm{~Pa}$ ), confirming the soft nature of these gels also reported in other studies ( $G^{\prime}$ lower than $10 \mathrm{~Pa}$ for similar PL formulations) ${ }^{31}$, for formulations containing $\mathrm{CNC}, \mathrm{G}^{\prime}$ increases by up to an impressive two orders of magnitude (1256 $\pm 82 \mathrm{~Pa}$ in PL-CNC 0.61). We next explored the nanoscale mechanical properties of the nanocomposite hydrogels to better understand and anticipate the possible interactions with cells at this size scale (Fig. 2-C and D). High CNC loadings increased hydrogel rigidity, as observed in the bulk rheological characterization (Figure 2-C). Strikingly, PL-CNC 0 , 0.31 and 0.61 fibers achieved elasticity values (Young's modulus) close to brain (0.1-1 kPa), muscle (8-17 $\mathrm{kPa}$ ) and collagenous bone (25-40 kPa) tissues, respectively ${ }^{43,44}$, Figure 2-D. However, whereas the rigidity of $\mathrm{PL}-\mathrm{CNC} 0$ hydrogel $(1.8 \pm 0.03 \mathrm{kPa})$ was similar to the values obtained along its fibers $(1.4 \pm 0.7 \mathrm{kPa})$ as demonstrated by the homogeneous elastic modulus along these samples (Figure 2-C), increase of CNC loadings is accompanied by an increase of fiber thickness (topography images) and sample heterogeneity (moduli images), showing significant difference between fiber ( $27 \pm 12 \mathrm{kPa}$ ) and hydrogel moduli (12 $\pm 7 \mathrm{kPa}$ ). Interestingly, it has been previously demonstrated that the fiber elastic modulus strongly decreases with increasing fiber diameter in either plasma or purified fibrinogen clots $^{39}$. It has been suggested that this negative diameter dependence of the modulus is correlated with a decrease of cross-sectional density of bonds within fibrin fibers with larger diameters. Moreover, is has also been demonstrated that clots with thinner fibers have higher whole-clot moduli due to the increased modulus of the single fibers and network branch points ${ }^{39,45}$. Remarkably, the mechanical properties of our nanocomposite hydrogels show a reverse trend. Increasing CNC loadings leads to: 1 ) thicker fibers with higher stiffness; 2) hydrogels with higher interfiber space (porosity), and thus lower branch points, with higher bulk stiffness. Therefore, these results further support the hypothesis that CNC might indeed increase the crosslinking density among protofibrils within the fibrin fiber in a process that also promotes the effective stress transfer from the protein matrix to the stiff reinforcing nanofiller. All these effects synergistically contribute to increase the fiber and hydrogel stiffness with high CNC content, demonstrating how the proposed strategy might be used to control the physical properties of PL hydrogels as biomaterials for TERM applications. 
Fibrillar nanocomposite hydrogels modulated matrix degradation and the proteins release profile. Besides poor mechanical properties, the use of fibrin based gels has been challenging due to their low in vitro/in vivo stability ${ }^{13}$. The use of fibrinolysis inhibitors such as aprotinin has been applied to mitigate this problem ${ }^{31}{ }^{46}$. However, it has been recently shown that aprotinin impaired vascular network formation in in vitro co-cultures of human umbilical vascular endothelial cells and human adipose-derived stem cells (hASCs) encapsulated in fibrin gels ${ }^{47}$, suggesting that its use might have negative implications in general tissue engineering strategies. Herein we did not use protease inhibitors, and therefore PL-CNC 0 hydrogels showed a markedly higher weight loss (95 $\pm 4 \%$ ) in comparison with PL-CNC $0.61(51 \pm 7 \%)$ after 7 days of degradation in phosphate buffered saline (PBS) (Figure 2-E) and the same trend was observed on total protein release (see supplementary information Figure S3). PL-CNC 0 was almost completely degraded over time, whereas PL-CNC 0.61 maintained $61 \%$ of the hydrogel protein at the end of the assay, demonstrating that CNC incorporation hinders the inherent proteolytic degradation of PL hydrogels and could therefore be an interesting alternative to the use of aprotinin.

Another important drawback associated with PL-based gels is the lack of control over dose and spatio-temporal delivery of specific platelet-derived biomolecules due to the low stability of the gel fibrillar matrix ${ }^{9,10}$. In an in vivo scenario, fibrin(ogen) is known for its ability to bind GFs through its heparin-binding domain and uses this affinity based mechanism to control the sequestering and subsequent release of signaling biomolecules ${ }^{48}$. For example, whereas fibroblast GF-2 (FGF-2) has high binding affinity to fibrinogen and strong retention in the fibrin matrix, PDGF-BB, the major GF constituent of platelet releasate ${ }^{49}$, has low binding affinity to fibrinogen and low retention in the fibrin matrix ( $>80 \%$ released after one day from fibrin gels) ${ }^{48}$. Considering the low affinity of PDGF-BB to fibrin, we select it as a GF model to investigate the potential impact of $\mathrm{CNC}$ on the sequestering and release profile of signaling biomolecules from nanocomposite hydrogels. Strikingly, whereas PL-CNC 0 showed a burst release profile of PDGF-BB within the first day (Figure 2-F), a behavior also observed in other PL based gels $^{17}$, in the nanocomposite hydrogels its release was below the detection limit of the ELISA assay. To guarantee that PDGF-BB release from the nanocomposite hydrogels was not detected do to experimental inaccuracies, we qualitatively evaluated by western blot the amount of PDGF-BB within the remaining hydrogels at the end of the assay (Figure 2-G). As expected, PDGF-BB was detectable in the nanocomposite formulations, indicating that this GF was sequestered within the hydrogel network, but not in PL-CNC 0 . The electrostatic binding of PDGF-BB (isoelectric point at $\mathrm{pH}$ 9.39) on the negatively charged CNC surface certainly contributes to its significant retention within the nanocomposite hydrogels, although other binding mechanisms might justify these results. Upon contact with platelet-derived biomolecules, a "hard" corona composed by high affinity proteins will develop on CNC surface over time, which might be stabilized by imine bonding. In theory, the recruitment of specific biomolecules to the nanoparticle surface might be advantageously exploited to modulate the protein corona composition and consequently the biological identity of the nanocomposite hydrogel microenvironment ${ }^{50}$. This concept would find a parallelism with the events happening during wound healing, where signaling molecules are released from ECM through finelyorchestrated mechanisms regulated by its binding affinity to matrix components and on the action of proteases ${ }^{27}$. Sulfated glycosaminoglycans (GAGs) in particular play important roles on the regulation of their spatio-temporal presentation within the wound microenvironment ${ }^{51}$. Considering that majority of CNC surface chemistry has high similarities with sulfated GAGs, it is plausible to hypothesize that it might favor the dynamic adsorption of biomolecules with high affinity to these polysaccharides ${ }^{52}$, including, not only PDGF-BB, but several other platelet-derived GF that establish specific binding to sulfated $\mathrm{GAGs}^{51}$. However, in contrast to GAGs the soluble form of cellulose sulfate derivatives, which are relatively flexible and predominantly hydrophilic polymers, the surface chemistry of rigid CNC is not isotropic and contains hydrophilic but also exposed hydrophobic domains attributed to the $(200) \beta /(220) \alpha$ crystalline edge of the cellulosic crystals, conferring them an amphiphilic character ${ }^{53}$. The recent developments on the characterization of bio-nano interfaces ${ }^{30,50}$ suggests that such structural and chemical anisotropy will certainly have an important impact on the entity of the protein corona built on CNC surface in the complex PL environment. Therefore, the nature of the interactions establish between specific $\mathrm{PL}$ biomolecules of interest with the CNC surface should be systematically characterized in future studies.

It worth noting that the equilibrium established between the imine bond and its corresponding precursors in aqueous media, namely, carbonyls of CNC and amines of PL's proteins, has a dynamic nature. Imines can participate in multiple types of equilibrium-controlled reactions (hydrolysis, exchange or metathesis) influenced by several external factors (e.g. precursor's concentration, $\mathrm{pH}$ or temperature $)^{54,55}$ which have been extensively explored in the development of controlled drug delivery systems ${ }^{56-}$ 58 . Although the carbonyls of CNC will preferentially react with the higher concentrated proteins in the $\mathrm{PL}$, e.g. albumin and fibrin(ogen) ${ }^{10}$, it can also react with GFs, representing therefore an additional level of control in the sequestration and release of these bioactive molecules from the nanocomposite hydrogel matrix. For example, it is know that the $\mathrm{pH}$ milieu in wounds can vary from acidic to basic depending on several factors, including the time course and wound-stage (acid in acute wounds and basic in chronic wounds $)^{59}$. Therefore, it is likely that the shift of the imine reaction equilibrium toward the precursors or the products in acute or chronic wounds, respectively, will result in different GF release profiles from the nanocomposite hydrogel matrix.

Stem cells were able to sense and respond to fibrillar hydrogels stiffness. As mentioned above, CNC incorporation improved PL hydrogel mechanical properties and stability due to the inherent CNC stiffness and the increased crosslinking density of the fibrillar hydrogel network. Having fiber diameters close to collagen fibrils 
(100-200 nm), these biomaterials closely mimic the topography and hierarchical structure of native ECM, characteristics that are not present in the typical polymer hydrogel systems used in TERM applications $^{60}$. The geometrical properties (e.g. porosity, fiber likearchitecture) of PL-CNC nanocomposites are crucial not only to allow sufficient nutrient and waste product transport ${ }^{61}$ and consequently enable cell survival, but also affect other important cell behaviors such as migration ${ }^{62,63}$. Acknowledging that dynamic changes in ECM microenvironment (e.g. mechanical cues and remodeling) have been shown to regulate cell functions ${ }^{44,64}$, we analyzed the impact of hydrogel features on stem cell supportive properties using hASCs. These cells are harvested from adipose tissue in large numbers and have been studied as an excellent and widely available autologous multipotent cell source ${ }^{65}$. The facile crosslinking process allowed hASCs encapsulation within the hydrogel network, showing high viability immediately after extrusion that was maintained in the following 7 days of culture (Figure 3-A). Although a higher cell density was visible on PL-CNC 0, the decrease of normalized metabolic activity indicates that this cell number is due to the extensive retraction of fibrin network leading the high cellular concentration in the gel volume (Figure 3-B). Upon hASCs encapsulation, PL-CNC 0 had a manifold densification of the fibrin network, referred to as clot retraction, which led to a dramatic $75 \%$ reduction in hydrogel diameter (Figure 3-C and D). In vivo, it is assumed that clot retraction plays a role in approximating the edges of a tissue defect and in concentrating the clot in the injured area ${ }^{66}$. However, this high contractile effect is undesirable for hydrogels to be applied in space filling of wounded sites. With CNC incorporation, the hydrogels showed a lower clot retraction accompanied by a higher proliferative capacity. The produced fibrin fiber network recapitulating the cellular-ECM interactions complexity and supporting cell adhesion and remodeling ${ }^{14}$ is clearly favored by CNC reinforcement.

Material's biophysical cues, namely topography and mechanical properties, are key parameters in the regulation of stem cell behavior $^{44,63}$. Regarding mechanical properties in particular, there is an increasing body of evidence supporting the concept that mesenchymal stem cells are extremely sensitive to tissue-level elasticity and that these physical cues are transduced into lineage specification $^{43,67}$. Therefore, considering the significantly different physicochemical properties of the developed nanocomposite hydrogels, we evaluated its impact on cell cytoskeleton organization, spreading and gene expression as a preliminary indication of stem cell preferential differentiation pathways.

In the soft PL gels, actin filaments were not well developed and visible, but these progressively became longer and organized on the stiffer nanocomposite hydrogels (Figure 4-A). 24 hours upon encapsulation, the majority of hASCS in PL-CNC 0 showed a round morphology that was even more evident at day 3 (Figure 4-C), mainly due to the contraction of the fibrin network in response to the pulling forces exerted by cells during culture (Figure 4-A). Conversely, PL-CNC 0.31 and 0.46 formulations showed high cell spreading area and cell axial ratio (Figure 4-B), demonstrating that the nanocomposite fibrillar network can counteract cell puling forces without undergoing extensive retraction. In PL-CNC 0.61 it was even possible to quantify these cellular parameters at a later time point (day 3 ) due to the minimal cellular overlap, showing that as cells continue to proliferate, they undergo high cytoskeleton elongation and present extended cell spreading area (Figure 4-D). This trend was confirmed by histological analysis for longer culture periods (14 days), demonstrating that whereas PL-CNC 0 show a highly compact cellularized structure due to the extensive hydrogel retraction, CNC-loaded hydrogels promote cell spreading and the formation of well-developed cellular networks (Figure S5). To evaluate the impact of these different cellular behaviors on ECM synthesis, we further analyzed the deposition of collagen type III by encapsulated hASCs, which is deposited during the initial phases of wound healing to provide a provisional cell supportive matrix ${ }^{2}$, being remodeled to other collagen types in later stages of wound repair. Interestingly, cell encapsulated in CNC-loaded hydrogels showed higher deposition of collagen type III and with a more fibrillar-like appearance than PL-CNC 0 hydrogels (Figure 4-E), suggesting that those formulations favor the progressive remodeling of the hydrogel 3D space with newly synthesized cellorigin ECM, a factor that might contribute to the increased hydrogel structural stability observed during cell culture. The marked differences observed in cell morphology also feedback the role of the hydrogel biophysical cues on the cells mechanotransduction process, since the stiffer fibrillar nanocomposite hydrogels promoted molecular mechanosensing mechanisms mediated by integrins which are transduced into actin polymerization and focal adhesion maturation ${ }^{63}$. Ultimately, the traction forces between hydrogel matrix and cells can alter gene expression, and therefore induce cell commitment toward different phenotypes ${ }^{68}$. In fact, gene expression analysis revealed that on one hand, hASCs laden on the softest PL CNC 0 hydrogels ( $2.2 \mathrm{kPa}$ ) showed an upregulation of transcription factors related to distinct lineages osteogenic ${ }^{69}$, tenogenic ${ }^{70}$, chondrogenic ${ }^{71}$ and adipogenic ${ }^{72}$ ), and PDGF-BB, an anti-morphogenic marker (Figure 5-A). These results are in agreement with the increasing use of PL as a prevailing supplement for cell culture in replacement of animal serum, being capable of faster in vitro stem cell expansion while maintaining their stemness $^{73-75}$, therefore suggesting its potential to repress cell differentiation into specific lineages. The presence of multiple cytokines and chemokines with opposite biological signaling roles in $\mathrm{PL}$ formulations might contribute to explain these results and highlight one of main difficulties generally associated with the use of blood derivatives in regenerative medicine: the establishment of rational cell stimuli protocols and consequently, therapeutic strategies $^{10}$. On the other hand, hASCs cultured on CNC-loaded hydrogels exhibited distinct genetic profiles, showing an upregulation osteogenic and chondrogenic related markers along with downregulation of lipoprotein lipase (LPL), an adipogenic marker, indicating potential cell commitment toward different lineages depending on CNC content ${ }^{76,77}$. In the stiffer PL-CNC 0.61 hydrogels, the levels of RUNX2 increased over time (from $11 \pm 1.1 \%$ at day 1 to $20 \pm 3.6 \%$ at day 7 ), while the expression of all the other studied transcription factors decreased. Furthermore, cells on this 
hydrogel formulation showed around 10-fold upregulation of alkaline phosphatase (ALP) expression, confirmed by increased ALP activity (Figure 5-B), indicating potential osteogenic commitment. Differently, on moderately stiff PL-CNC 0.31 hydrogels, from day 1 to day 7 , hASCs show an increased expression of two chondrogenic related markers namely, cartilage oligomeric matrix protein (COMP) and SRY-box 9 (SOX9). Remarkably, although the use of PL-based gels has attracted significant interest in the promotion of vascularization in regenerative strategies due to their contents in angiogenic GFs that favor mesenchymal stem cells invasion and endothelial sprouting ${ }^{17,31}$, we observed that the expression of angiogenic related genes such as vascular endothelial GF (VEGF) declined more than one fold at day 7 in PL-CNC 0 (Figure 4-A). In contrast, in the moderately stiff matrices PL-CNC 0.31, the expression of this gene increased to the levels of control group (PLCNC 0 at day 1 ). These findings suggest that, as in native $\mathrm{ECM}^{78}$, an adequate physical and chemical environment capable of sequestering and presenting angiogens to cell receptors might be needed to sustain the angiogenic potential of PL over time. Therefore, the interaction between angiogens and nanocomposite hydrogel matrix and how these synergies can potentiate vascularization processes deserve further study.

In summary, we developed a method to create injectable fibrillar nanocomposite hydrogels based on PL combined with CNC as a platform that allowed the use of this blood derivative not only as a source of signaling biomolecules involved in wound healing, but also as an injectable cell carrier biomaterial with tunable biophysical and biochemical cues for cell delivery in TERM strategies. We have demonstrated that by increasing CNC loading in natural fibrillar PL hydrogels it is possible to increase fiber diameter, stiffness, interfibrillar porosity, hinder the typical fast clot degradation, and improve bioactive molecules sequestering, enabling therefore its use as effective ECM-mimetic biomaterials in which we can control the 3D cell microenvironment (Scheme 2). The physical changes occurring in the fibrillar network stemming from CNC load and crosslinking led to hydrogels that do not compromise the outstanding cytocompatibility of $\mathrm{PL}$ gels, can resist extensive retraction upon 3D cell culture and have a marked impact on determining cell behavior. We have shown that cell cytoskeletal organization can be easily manipulated by controlling CNC loadings and these effects might be adjusted to direct the fate of encapsulated hASCs toward specific lineages.

\section{Conclusions}

We have developed in this study injectable PL nanocomposite fibrillar hydrogels that are biochemically, mechanically and structurally tunable, enabling the modulation of multiple parameters of the 3D cell microenvironment in ways that are poorly achieved with other traditional polymer-based strategies. It is anticipated that this natural-based hydrogel platform will find multiple applications as a bioactive cell carrier matrix capable of harnessing the regenerative potential of platelets content and promote regenerative wound healing outcomes in tissue engineering strategies.

\section{Conflicts of interest}

There are no conflicts to declare.

\section{Acknowledgements}

The authors thank Hospital da Prelada (Porto, Portugal) for providing adipose tissue samples. Authors acknowledge the financial support from project Recognize (UTAP-ICDT/CTMBIO/0023/2014), project NORTE-01-0145-FEDER- 000021 supported by Norte Portugal Regional Operational Programme (NORTE 2020), under the PORTUGAL 2020 Partnership Agreement, through the European Regional Development Fund (ERDF); the European Union Framework Programme for Research and Innovation HORIZON 2020, under the TEAMING Grant agreement No 739572 - The Discoveries CTR EU, Forecast 668983, Marie Skłodowska-Curie grant agreement No 706996 and CHEM2NATURE 692333; FCT/MCTES (Fundação para a Ciência e a Tecnologia/ Ministério da Ciência, Tecnologia, e Ensino Superior) and the Fundo Social Europeu através do Programa Operacional do Capital Humano (FSE/POCH) in the framework of PhD grant PD/59/2013 $\mathrm{PD} / \mathrm{BD} / 113807 / 2015$ for BBM, Post-Doc grant SFRH/BPD/112459/2015 for R.D.

\section{Notes and references}

Supporting Information. Materials and methods details. Morphological and chemical characterization of modified CNC and additional physical hydrogel characterization.

* Corresponding author: email: megomes@i3bs.uminho.pt, rui.domingues@i3bs.uminho.pt

1. S. A. Eming, P. Martin and M. Tomic-Canic, Science Translational Medicine, 2014, 6, $265 s r 266$.

2. G. C. Gurtner, S. Werner, Y. Barrandon and M. T. Longaker, Nature, 2008, 453, 314-321.

3. S. Padilla, M. Sánchez, G. Orive and E. Anitua, Trends in Biotechnology, 2017, 35, 192-202.

$4 . \quad$ D. M. Dohan Ehrenfest, L. Rasmusson and T. Albrektsson, Trends in Biotechnology, 2009, 27, 158-167.

5. T. Burnouf, H. A. Goubran, T.-M. Chen, K.-L. Ou, M. ElEkiaby and M. Radosevic, Blood Reviews, 2013, 27, 77-89.

6. Y. Qian, Q. Han, W. Chen, J. Song, X. Zhao, Y. Ouyang, W. Yuan and C. Fan, Frontiers in Chemistry, 2017, 5, 1-8.

7. R. Crespo-Diaz, A. Behfar, G. W. Butler, D. J. Padley, M. G. Sarr, J. Bartunek, A. B. Dietz and A. Terzic, Cell Transplantation, 2011, 20, 797-811.

8. N. Fekete, M. Gadelorge, D. Fürst, C. Maurer, J. Dausend, S. Fleury-Cappellesso, V. Mailänder, R. Lotfi, A. Ignatius, L. Sensebé, P. Bourin, H. Schrezenmeier and M. T. Rojewski, Cytotherapy, 2012, 14, 540-554.

9. P. S. Babo, R. L. Reis and M. E. Gomes, Journal of Materials Chemistry B, 2017, 5, 3617-3628. 
10. B. B. Mendes, M. Gomez-Florit, P. S. Babo, R. L. Reis, R. M. A. Domingues and M. E. Gomes, Advanced drug delivery reviews, 2017, DOI: 10.1016/j.addr.2017.12.018.

11. D. E. Roberts, A. McNicol and R. Bose, Journal of Biological Chemistry, 2004, 279, 19421-19430.

12. J. M. Pachence, Journal of Biomedical Materials Research Part A, 1996, 33, 35-40.

13.

A. C. Brown and T. H. Barker, Acta Biomaterialia, 2014, 10, 1502-1514.

14. A. M. Rosales and K. S. Anseth, Nature reviews. Materials, 2016, 1, 1-15.

15. H.-L. Wang and G. Avila, European Journal of Dentistry, 2007, 1, 192-194.

16.

M. P. Hall, J. P. Ward and D. A. Cardone, Bulletin of the Hospital for Joint Disease, 2013, 71, 54-59.

17. T. M. Fortunato, C. Beltrami, C. Emanueli, P. A. De Bank and G. Pula, Scientific Reports, 2016, 6, 1-15.

18. M. Sadeghi-Ataabadi, Z. Mostafavi-pour, Z. Vojdani, M. Sani, M. Latifi and T. Talaei-Khozani, Materials Science and Engineering: C, 2017, 71, 372-380.

19. S. T. Robinson and L. P. Brewster, in Physiologic and Pathologic Angiogenesis - Signaling Mechanisms and Targeted Therapy, eds. D. Simionescu and A. Simionescu, InTech, Rijeka, 2017, DOI: 10.5772/66934, pp. 365-383.

20. A. Motealleh and N. S. Kehr, Advanced Healthcare Materials, 2017, 6, 1-19.

21. P. Schexnailder and G. Schmidt, Colloid and Polymer Science, 2009, 287, 1-11.

22. M. Adnan, A. A. Hani, M. A. Hussain, A. Musab, N. Fozia Al, A.-H. Faten, O. Rahmi and K. Ali, Biomedical Materials, 2016, 11, 1-15.

23.

R. M. A. Domingues, M. E. Gomes and R. L. Reis, Biomacromolecules, 2014, 15, 2327-2346.

24. R. M. A. Domingues, M. Silva, P. Gershovich, S. Betta, P. Babo, S. G. Caridade, J. F. Mano, A. Motta, R. L. Reis and M. E. Gomes, Bioconjugate chemistry, 2015, 26, 15711581.

25. K. J. De France, T. Hoare and E. D. Cranston, Chemistry of Materials, 2017, 29, 4609-4631.

26. A. C. Mitchell, P. S. Briquez, J. A. Hubbell and J. R. Cochran, Acta Biomaterialia, 2016, 30, 1-12.

27. P. S. Briquez, J. A. Hubbell and M. M. Martino, Advances in Wound Care, 2015, 4, 479-489.

28. Z. Kai, P. Dieter, B. Erica, G. Thomas and F. Steffen, Macromolecular Symposia, 2009, 280, 28-35.

29. D. Peschel, K. Zhang, N. Aggarwal, E. Brendler, S. Fischer and T. Groth, Acta Biomaterialia, 2010, 6, 2116-2125.

30. D. Peschel, K. Zhang, S. Fischer and T. Groth, Acta Biomaterialia, 2012, 8, 183-193.

31. S. T. Robinson, A. M. Douglas, T. Chadid, K. Kuo, A. Rajabalan, H. Li, I. B. Copland, T. H. Barker, J. Galipeau and L. P. Brewster, Acta Biomaterialia, 2016, 36, 86-98.

32. P. P. Carvalho, X. Wu, G. Yu, I. R. Dias, M. E. Gomes, R. L. Reis and J. M. Gimble, Cells, tissues, organs, 2011, 194, 494-500.

33. J. M. Gimble and F. Guilak, Current topics in developmental biology, 2003, 58, 137-160.

34. J. Gimble and F. Guilak, Cytotherapy, 2003, 5, 362-369.

35. M. Dominici, K. Le Blanc, I. Mueller, I. Slaper-Cortenbach, F. Marini, D. Krause, R. Deans, A. Keating, D. Prockop and E. Horwitz, Cytotherapy, 2006, 8, 315-317.

36.
M. W. Pfaffl, A. Tichopad, C. Prgomet and T. P. Neuvians, Biotechnology letters, 2004, 26, 509-515.

L. Huang, J. P.-L. Hsiao, C. Powierza, R. M. Taylor and S. T. Lord, Biochemistry, 2014, 53, 7824-7834.

W. Li, J. Sigley, M. Pieters, C. C. Helms, C. Nagaswami, J. W. Weisel and M. Guthold, Biophysical Journal, 2016, 110, 1400-1410.

M. De Spirito, G. Arcovito, M. Papi, M. Rocco and F. Ferri, Journal of Applied Crystallography, 2003, 36, 636-641.

N. A. Kurniawan, J. Grimbergen, J. Koopman and G. H. Koenderink, Thrombosis and haemostasis, 2014, 12, 16871696.

C. Duval, P. Allan, S. D. Connell, V. C. Ridger, H. Philippou and R. A. Ariens, Thrombosis and haemostasis, 2014, 111, 842-850.

A. J. Engler, S. Sen, H. L. Sweeney and D. E. Discher, Cell, 2006, 126, 677-689.

K. H. Vining and D. J. Mooney, Nature Reviews Molecular Cell Biology, 2017, 18, 728-742.

E. A. Ryan, L. F. Mockros, J. W. Weisel and L. Lorand, Biophysical Journal, 1999, 77, 2813-2826.

P. S. Briquez, K. M. Lorentz, H. M. Larsson, P. Frey and J. A. Hubbell, Biomaterials, 2017, 135, 1-9.

S. Muhleder, K. Pill, M. Schaupper, K. Labuda, E. Priglinger, P. Hofbauer, V. Charwat, U. Marx, H. Redl and W. Holnthoner, Stem cell research \& therapy, 2018, 9, 113.

M. M. Martino, P. S. Briquez, A. Ranga, M. P. Lutolf and J. A. Hubbell, Proceedings of the National Academy of Sciences of the United States of America, 2013, 110, 45634568.

P. R. Amable, R. B. Carias, M. V. Teixeira, I. da Cruz Pacheco, R. J. Correa do Amaral, J. M. Granjeiro and R. Borojevic, Stem cell research \& therapy, 2013, 4, 1-13.

M. P. Monopoli, C. Aberg, A. Salvati and K. A. Dawson, Nature nanotechnology, 2012, 7, 779-786.

I. Capila and R. J. Linhardt, Angewandte Chemie, 2002, 41, 391-412.

P. C. Ke, S. Lin, W. J. Parak, T. P. Davis and F. Caruso, ACS nano, 2017, 11, 11773-11776.

I. Kalashnikova, H. Bizot, B. Cathala and I. Capron, Biomacromolecules, 2012, 13, 267-275.

R. W. Layer, Chemical reviews, 1963, 63, 489-510.

M. E. Belowich and J. F. Stoddart, Chemical Society reviews, 2012, 41, 2003-2024.

Y. Tao, S. Liu, Y. Zhang, Z. Chi and J. Xu, Polymer Chemistry, 2018, 9, 878-884.

A. Seoane, R. J. Brea, A. Fuertes, K. A. Podolsky and N. K. Devaraj, Journal of the American Chemical Society, 2018, 140, 8388-8391.

G. Zhao, L. Long, L. Zhang, M. Peng, T. Cui, X. Wen, X. Zhou, L. Sun and L. Che, Scientific Reports, 2017, 7, 3383.

L. A. Schneider, A. Korber, S. Grabbe and J. Dissemond, Archives of Dermatological Research, 2007, 298, 413-420.

T. Ushiki, Archives of histology and cytology, 2002, 65, 109-126.

Q. L. Loh and C. Choong, Tissue engineering. Part B, Reviews, 2013, 19, 485-502.

J. Demol, D. Lambrechts, L. Geris, J. Schrooten and H. Van Oosterwyck, Biomaterials, 2011, 32, 107-118.

Y. Li, Y. Xiao and C. Liu, Chemical reviews, 2017, 117, 4376-4421. 
64. W. L. Murphy, T. C. McDevitt and A. J. Engler, Nature materials, 2014, 13, 547-557.

65. T. Rada, R. L. Reis and M. E. Gomes, Tissue Engineering Part B: Reviews, 2009, 15, 113-125.

66. M. E. Carr, Jr. and S. L. Carr, Blood Coagulution Fibrinolysis, 1995, 6, 79-86.

67. A. W. Holle, J. L. Young, K. J. Van Vliet, R. D. Kamm, D. Discher, P. Janmey, J. P. Spatz and T. Saif, Nano Letters, 2018, 18, 1-8.

68. Enateri V. Alakpa, V. Jayawarna, A. Lampel, Karl V. Burgess, Christopher C. West, Sanne C. J. Bakker, S. Roy, N. Javid, S. Fleming, Dimitris A. Lamprou, J. Yang, A. Miller, Andrew J. Urquhart, Pim W. J. M. Frederix, Neil T. Hunt, B. Péault, Rein V. Ulijn and Matthew J. Dalby, Chem, 2016, 1, 298-319.

69. T. Komori, Frontiers Bioscience, 2008, 13, 898-903.

70. L. Gaut and D. Duprez, Wiley Interdisciplary Reviews Developmental Biology, 2016, 5, 5-23.

71. C. Healy, D. Uwanogho and P. T. Sharpe, Developmental Dynamics, 1999, 215, 69-78.

72. E. D. Rosen, C.-H. Hsu, X. Wang, S. Sakai, M. W. Freeman, F. J. Gonzalez and B. M. Spiegelman, Genes \& development, 2002, 16, 22-26.

73. E. Fernandez-Rebollo, B. Mentrup, R. Ebert, J. Franzen, G. Abagnale, T. Sieben, A. Ostrowska, P. Hoffmann, P.-F. Roux, B. Rath, M. Goodhardt, J.-M. Lemaitre, O. Bischof, F. Jakob and W. Wagner, Scientific Reports, 2017, 7, 5132.

74. T. Burnouf, D. Strunk, M. B. C. Koh and K. Schallmoser, Biomaterials, 2016, 76, 371-387.

75. S. M. Jonsdottir-Buch, R. Lieder and O. E. Sigurjonsson, PloS one, 2013, 8, e68984.

76. J. Upadhyay, O. M. Farr and C. S. Mantzoros, Metabolism, 2015, 64, 105-113.

77. C. Granéli, A. Thorfve, U. Ruetschi, H. Brisby, P. Thomsen, A. Lindahl and C. Karlsson, Stem cell research, 2014, 12, 153-165.

78. V. Vogel, Annual review of physiology, 2018, 80, 353-387. 


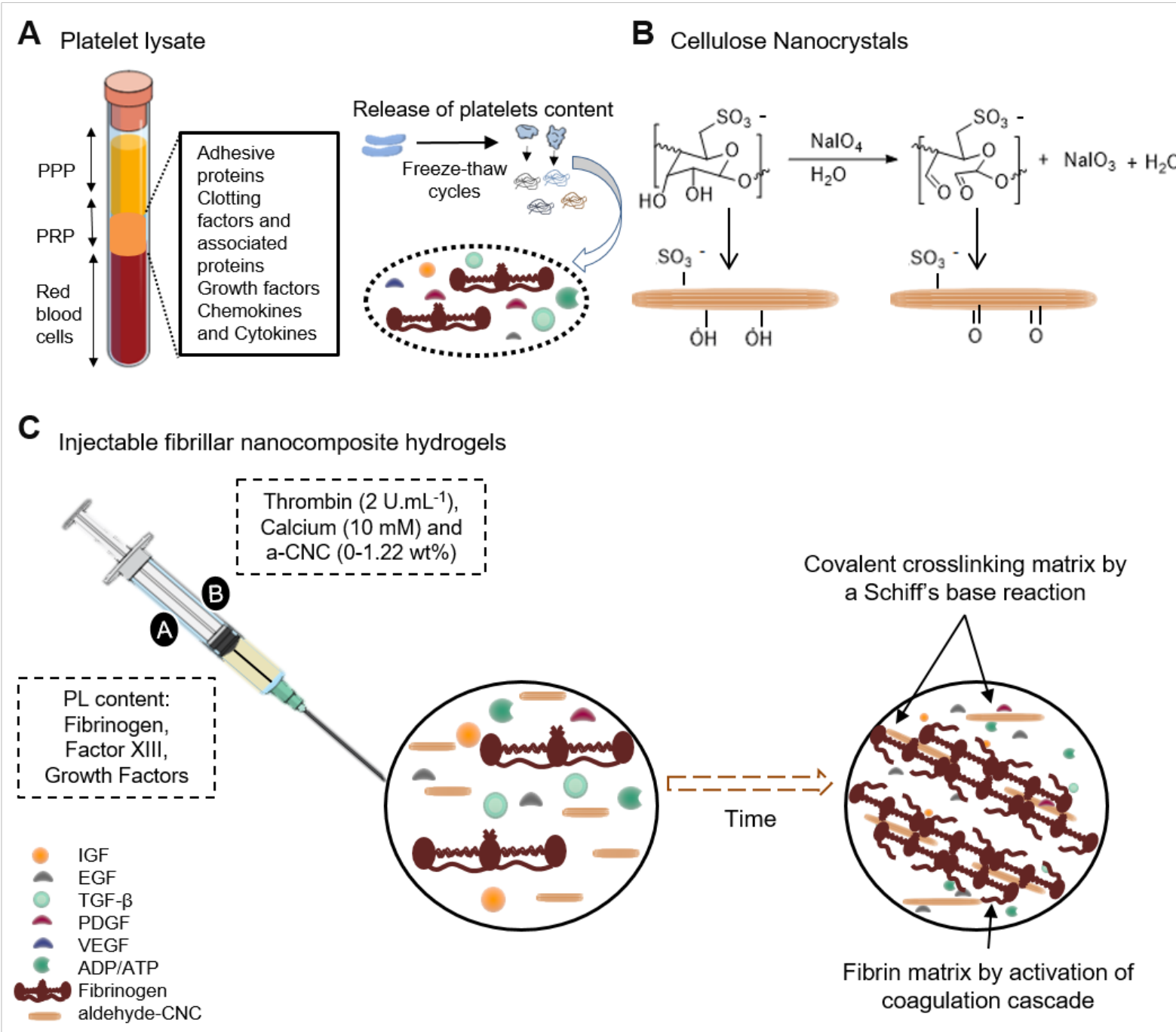

Scheme 1. Schematic representation of the preparation of natural fibrillar nanocomposite hydrogels (PL-CNC 00.61). PL was obtained by freeze-thaw cycles which allowed the release of platelets content (A). CNC were modified with aldehyde groups by periodate oxidation (B). Injectable fibrillar nanocomposite hydrogels were prepared using fibrin polymerization (coagulation cascade induction) and covalent crosslinking between aldehydes on CNC surface and amine groups of PL proteins (Schiff's base reaction) (C). 


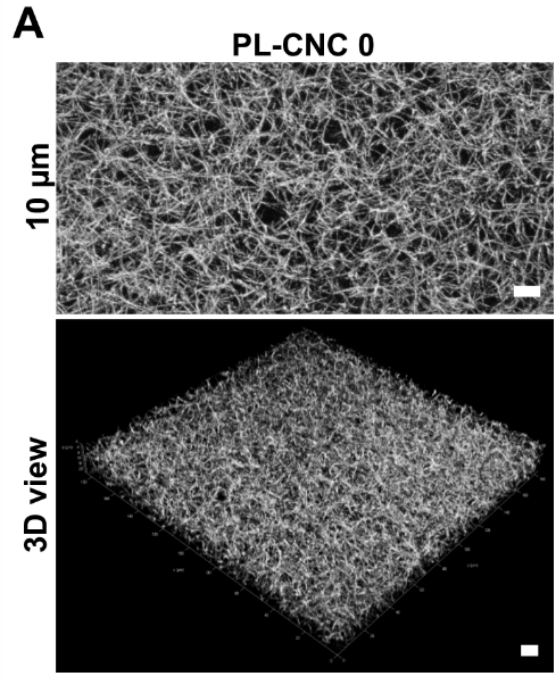

B

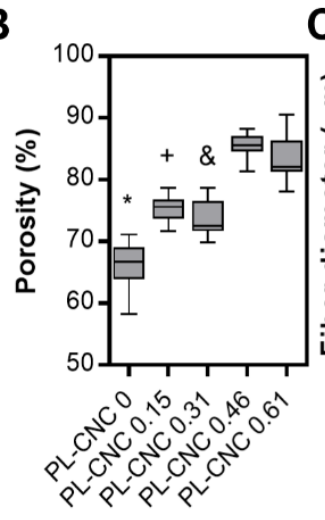

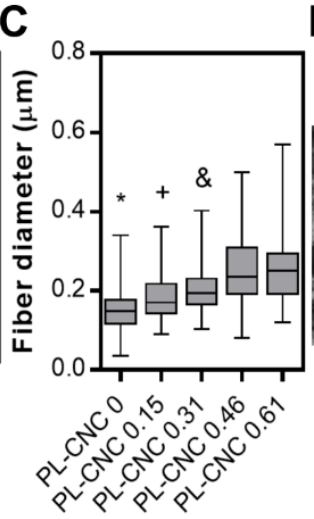

PL-CNC 0.31

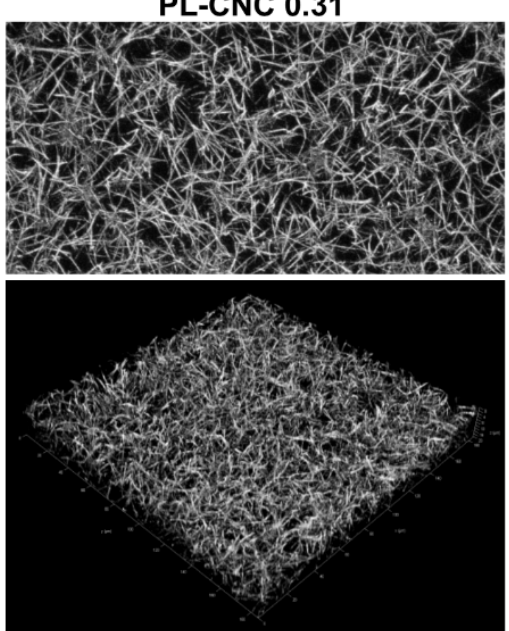

D
PL-CNC 0.61

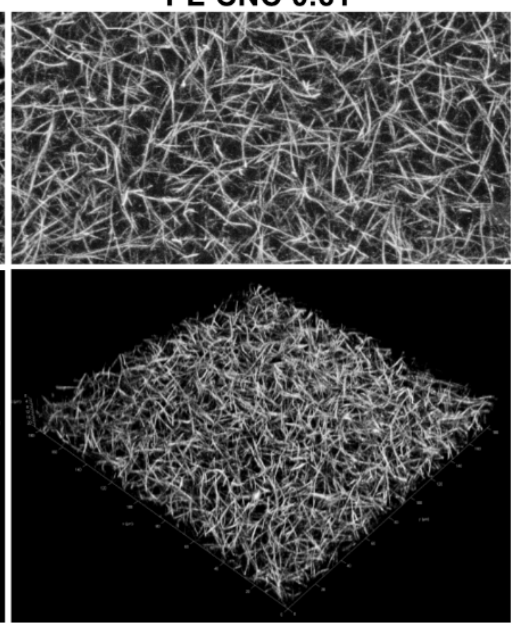

PL-CNC

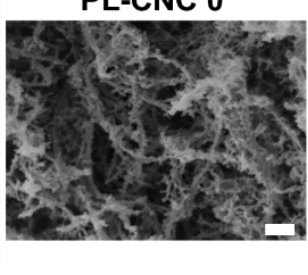

PL-CNC 0.15

PL-CNC 0.61

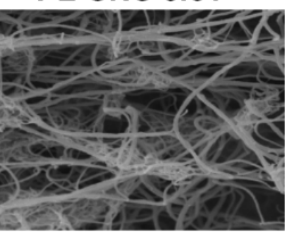

Figure 1. Microstructure of $\mathrm{PL}$ and nanocomposite fibrillar hydrogels. Maximum intensity projection (10 $\mu \mathrm{m}$ height stack) and 3D view of Alexa Fluor 488 conjugated fibrinogen showing fibrin polymerized network (A).

Percent of porosity of a $4 \mu \mathrm{m}$ thick section of fluorescent confocal images (B). Fiber diameter determined from SEM images (C). Microstructural analysis by SEM (D). * PL-CNC 0 vs PL-CNC (0.15-0.61); + PL-CNC 0.15 vs PL-CNC (0.46-0.61) and \& PL-CNC 0.31 vs PL-CNC (0.46-0.61). Scale bars= $10 \mu \mathrm{m}(\mathrm{A})$ and $1 \mu \mathrm{m}(\mathrm{D})$. 
A

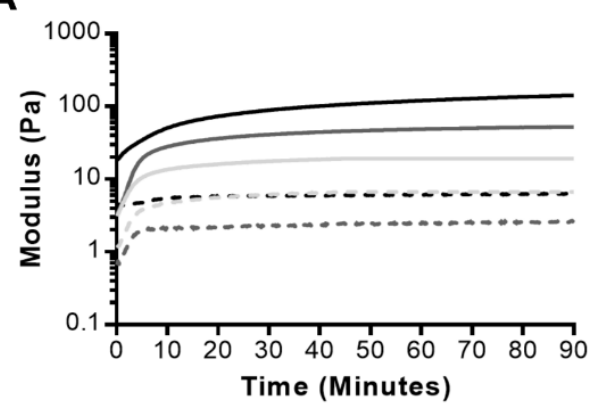

C

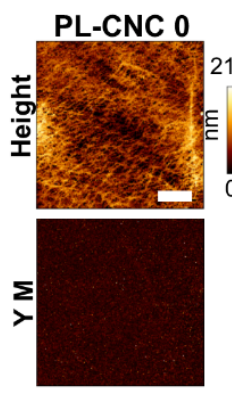

E

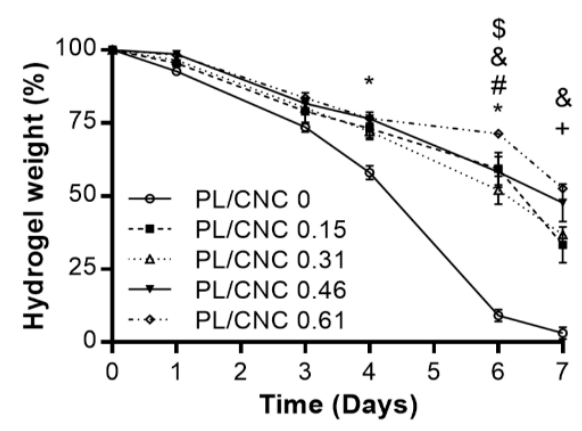

B

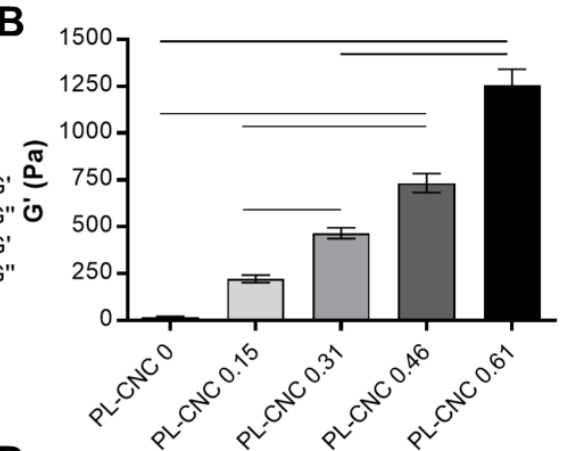

D
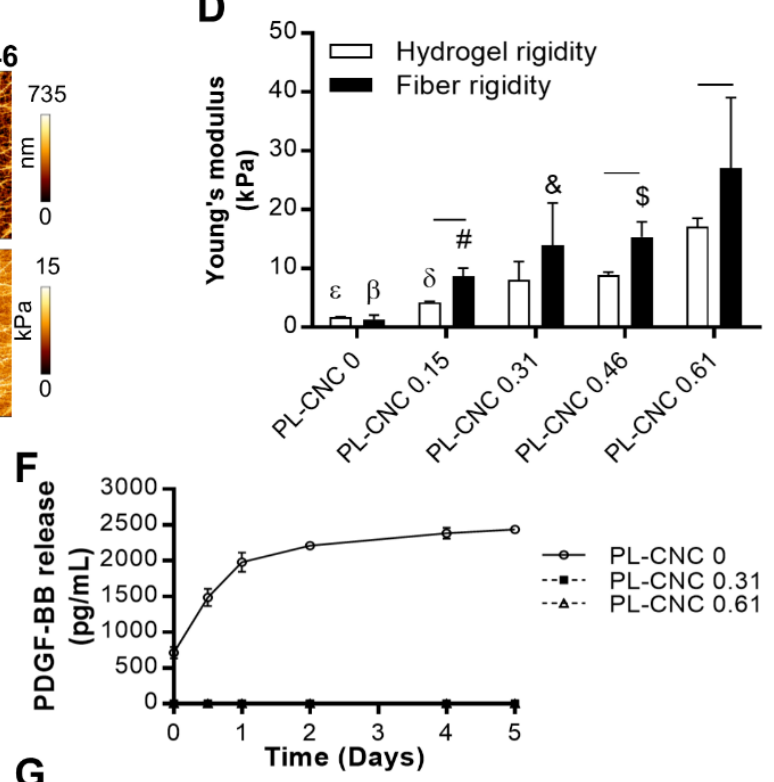

G

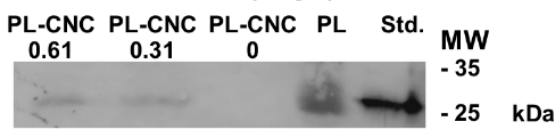

Figure 2. Physical characterization of nanocomposite hydrogels. Storage modulus ( $\left.G^{\prime}\right)$ versus polymerization and crosslinking time at $1 \%$ strain and frequency of $1 \mathrm{~Hz}(A)$. Storage modulus ( $\left.\mathrm{G}^{\prime}\right)$ of $\mathrm{PL} / \mathrm{CNC}$ hydrogels averaged from the frequency-sweep measurements between 0.1 to $1 \mathrm{~Hz}$ at $0.5 \%$ strain (B). Representative images of AFMgenerated height (top) and Young's modulus (YM) (bottom) for PL-CNC hydrogels conditions (C) and Average Young's modulus for hydrogels (all image) and fibrils in each PL-CNC formulation (D). Weight loss over 7 days immersed in PBS (E). Cumulative concentration release of PDGF-BB in cell culture medium (F). Western Blot of PDGF-BB within natural fibrillar hydrogels at day 5 in cell culture medium (G). - between selected conditions, * PL-CNC 0 vs PL-CNC (0.15-0.61); + PL-CNC 0.15 vs PL-CNC (0.46-0.61); \# PL-CNC 0.15 vs PL-CNC 0.61; \& PL-CNC 0.31 vs PL-CNC 0.61; \$ PL-CNC 0.46 vs PL-CNC 0.61. Hydrogel rigidity: $\varepsilon$ PL-CNC 0 vs PL-CNC 0.61; $\delta$ PL-CNC 0.15 vs 
PL-CNC 0.61; Fiber rigidity: $\beta$ PL-CNC 0 vs PL-CNC 0.61; \# PL-CNC 0.15 vs PL-CNC 0.61; \& PL-CNC 0.31 vs PL-CNC 0.61; \$ PL-CNC 0.46 vs PL-CNC 0.61. Scale bars $=2 \mu \mathrm{m}(\mathrm{C})$.

A
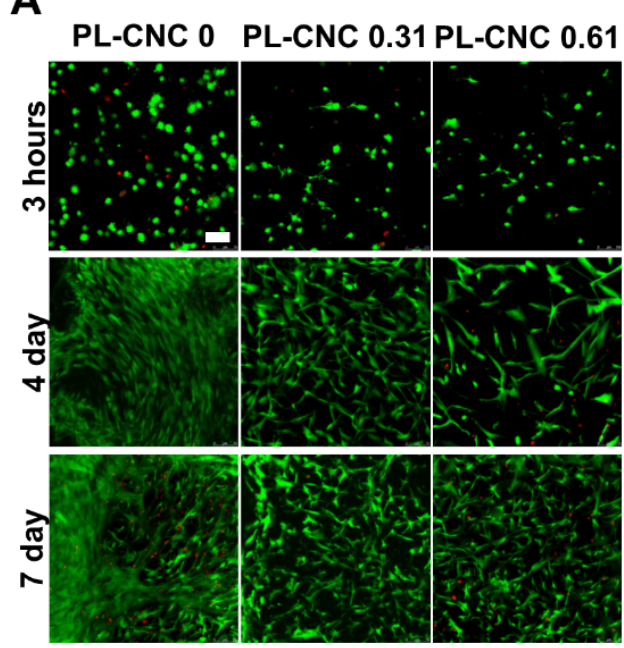

B
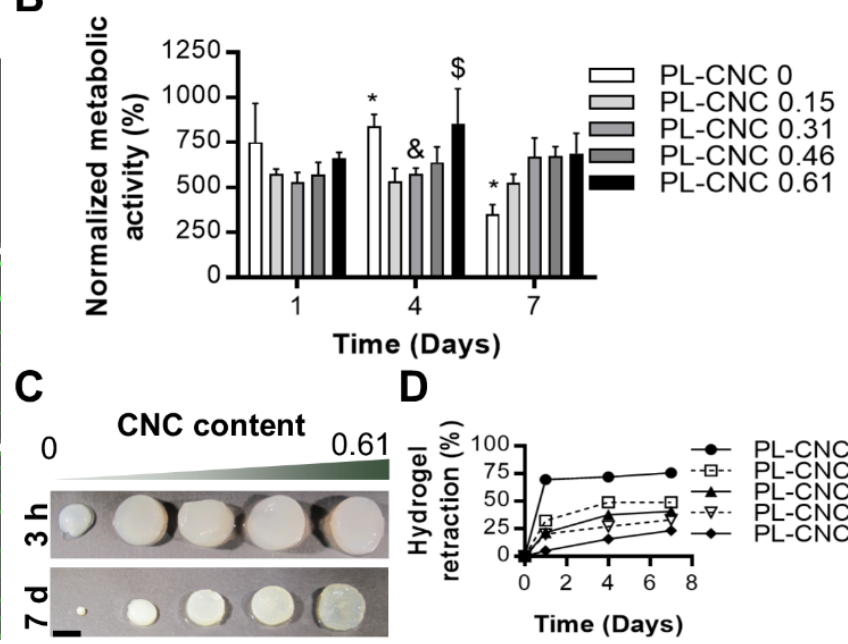

D

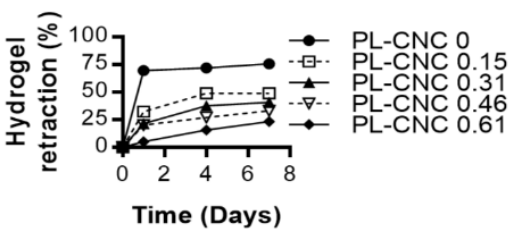

Figure 3. In vitro evaluation of cell supportive properties and hydrogels retraction upon cell encapsulation. Live/Dead staining with Calcein AM/PI (green: live cell; red: dead cell) of hASCs encapsulated in PL/CNC hydrogels (A). Metabolic activity normalized by total DNA content (B). Photographs of PL-CNC hydrogels after 3 hours and 7 days in culture (C). Hydrogels diameter retraction in percentage at 1, 4 and 7 days - statistically differences in all formulations, except PL-CNC 0.31 vs $P L-C N C 0.46$ at time point 1 (D). * PL-CNC 0 vs $P L-C N C$ (0.15-0.61); \& PL-CNC 0.31 vs PLCNC 0.61 and \$ PL-CNC 0.61 vs PL-CNC 0.15. Scale bars: $75 \mu \mathrm{m}(\mathrm{A})$ and $4 \mathrm{~mm}(C)$. 
A PL-CNC 0
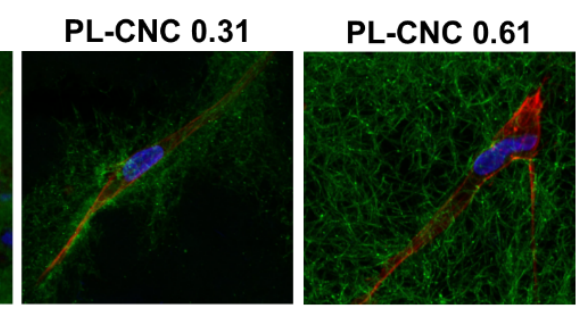

C
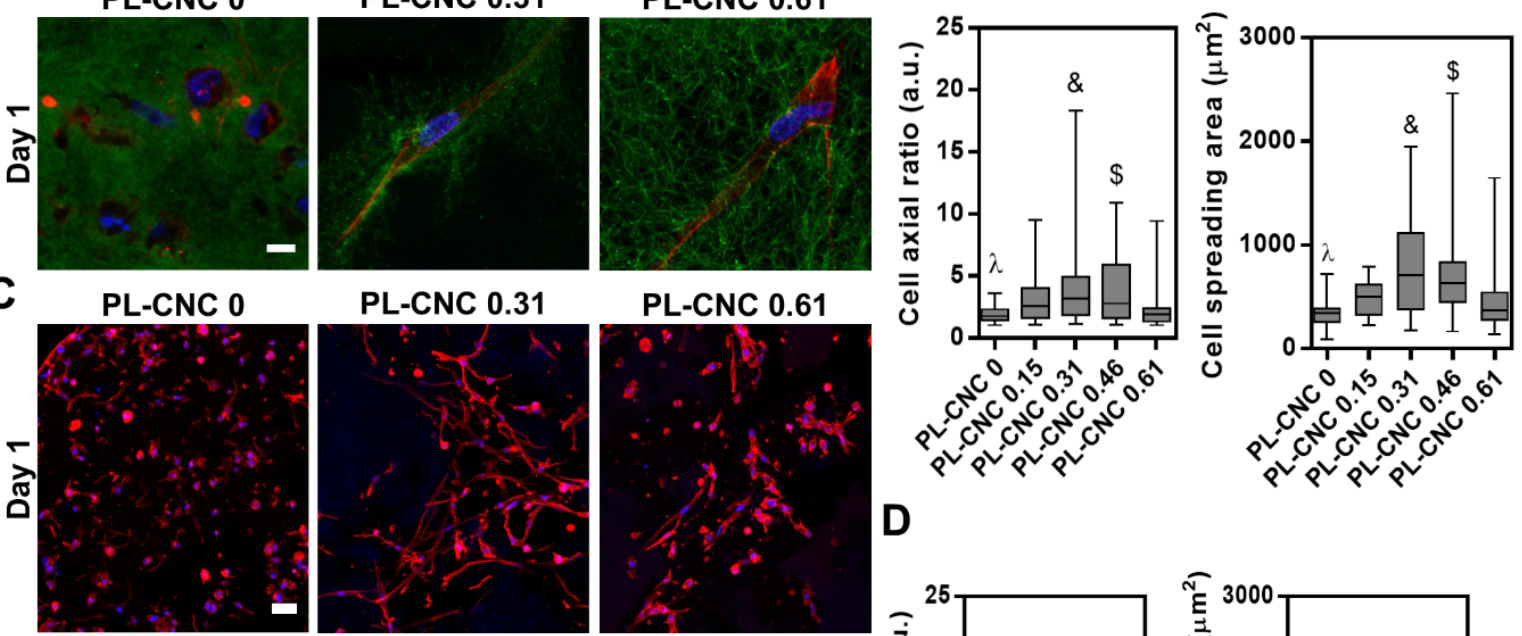

D
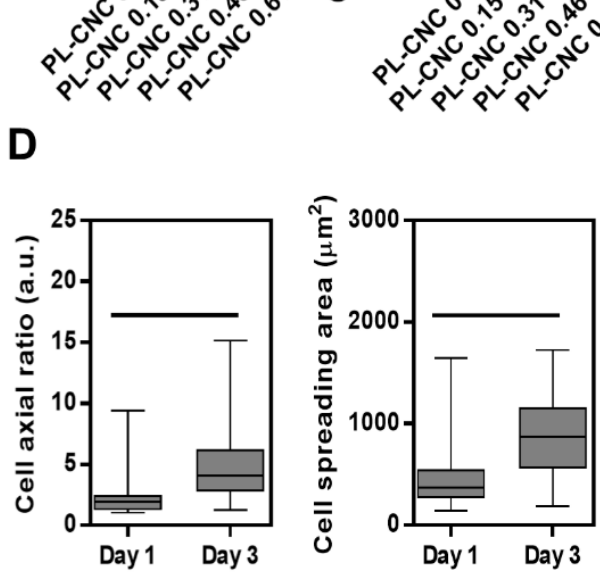

E
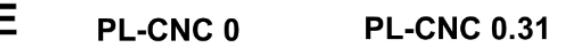

PL-CNC 0.61
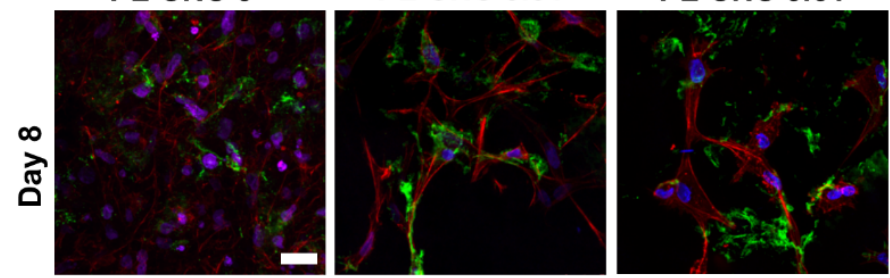

Figure 4. In vitro evaluation of cellular differentiation in PL and PL-CNC hydrogels. Fluorescence microscopy images showing cytoskeleton organization in the fibrin matrix after 1 day in culture (A). Cell axial ratio and cell spreading area after 1 day of culture were quantified for all conditions (B). Fluorescence microscopy images showing cytoskeleton organization after 1 and 3 days in culture $(C)$. Cell axial ratio and cell spreading area after 1 and 3 days of culture were quantified for PL-CNC 0.61 (D). Collagen Type III deposition after 8 days in culture. Staining fibrinogen (green) (A), collagen Type III (green) (E), actin (red) and nuclei (blue) (A, C and E). Scale bars: $10 \mu m(A)$ and $50 \mu m(C)$. between selected conditions; $\lambda$ PL-CNC 0 vs PL-CNC (0.15-0.46); \& PL-CNC 0.31 vs PL-CNC 0.61 and $\$ P L-C N C 0.46$ vs PL-CNC 0.61. 

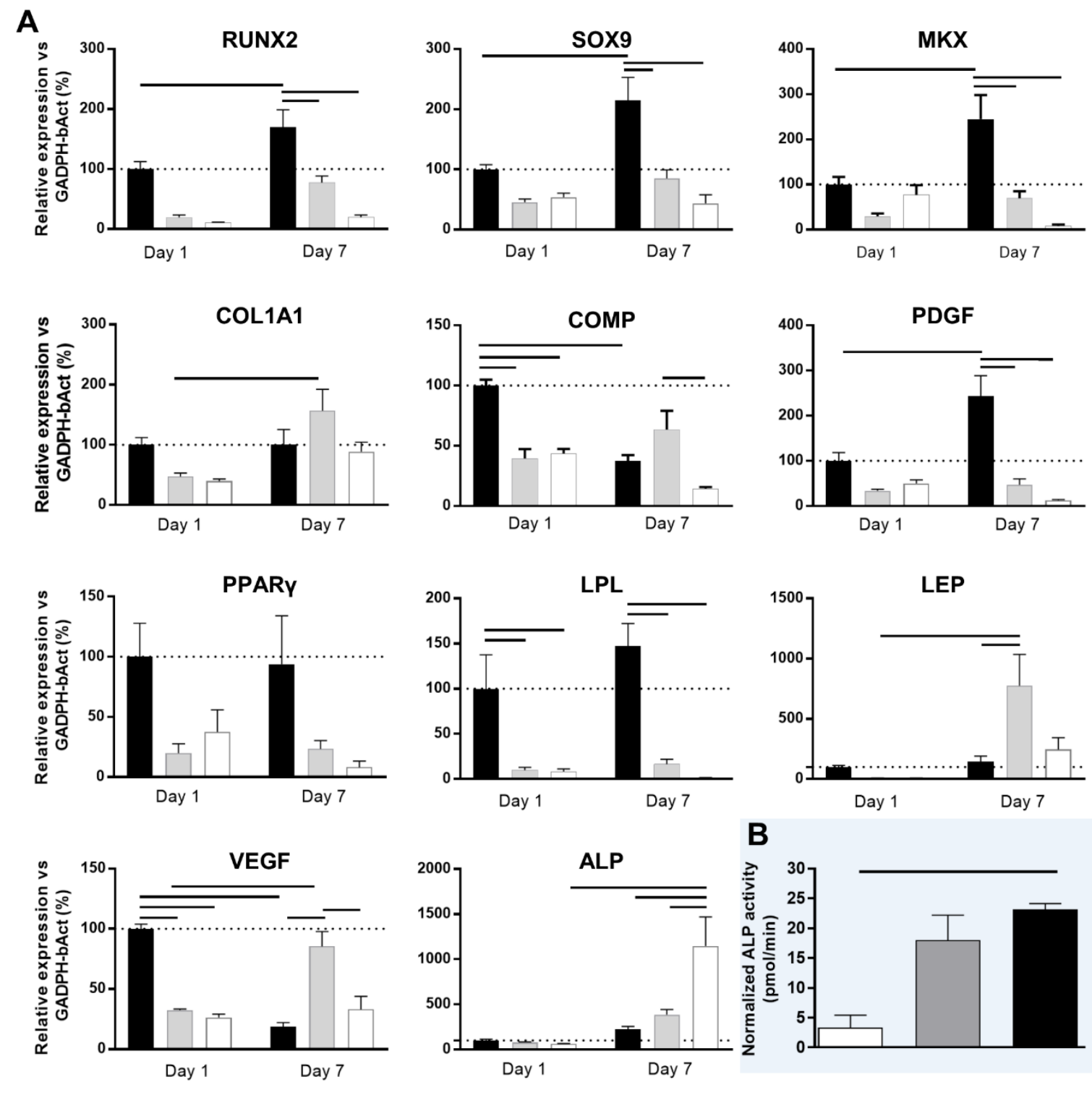

B

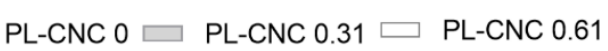

Figure 5. hASCs were assessed for the expression of chondrogenic (SOX-9 and COMP), osteogenic (RUNX2, COLA1 and ALP), adipogenic (LPL and PPAR ), tenogenic (MKX), anti-morphogenic (PDGF) and angiogenic markers (VEGF) (A). ALP activity was quantified after 9 days in culture (B). Runt-related transcription factor 2 (RUNX2), SRY-box 9 (SOX9), Mohawk (MKX), collagen type I alpha 1 chain (COLA1), cartilage oligomeric matrix protein (COMP), platelet-derived growth factor-B (PDGF), peroxisome proliferator-activated receptor $\gamma(P P A R \gamma)$, lipoprotein lipase (LPL), leptin (LEP), vascular 
endothelial growth factor (VEGFA) and alkaline phosphatase (ALP), and leptin (LEP). - between selected conditions.

A

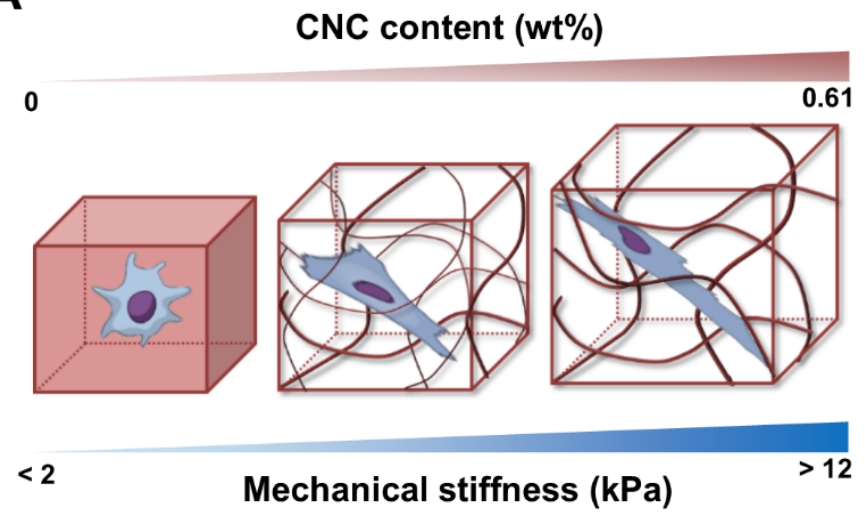

B CNC content (wt\%)

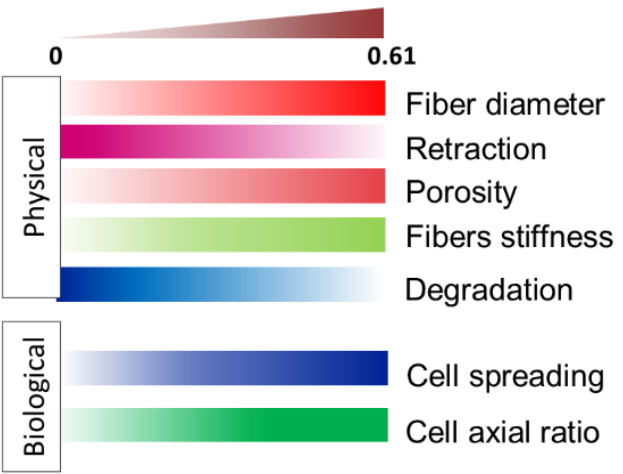

Scheme 2. Schematic representation of the cytoskeletal organization of encapsulated hASCs in function of PL nanocomposite hydrogel composition and stiffness (A). Influence of each studied parameter in the hydrogel physical properties and cell behavior (B). 
